\title{
Transmission Electron Microscopy of the Ebola virus
}

\author{
Daniel R. Beniac, Pasquale L. Melito, Steven M. Jones, and Tim F. Booth
}

National Microbiology Laboratory, 1015 Arlington Street, Winnipeg, Manitoba, R3E 3R2, Canada.

Ebola virus is a single-stranded RNA virus, it is a member of the family Filoviridae in which there are two genera, Ebolavirus and Marburgvirus. The genus Ebolavirus is divided further into five species, named by the locations from which they were first isolated (Zaire, Sudan, Reston, Cote d'Ivoire, Bundibugyo) [1,2]. Ebola virus causes hemorrhagic fever in humans, it stimulates the most severe form of the pathology and features the highest case fatality rate [3]. Ebola virus is classified as a 'Biosafety Level 4' agent (BSL-4, World Health Organization, BSL-4) [4]. The lack of theraputics, coupled with concerns about weaponization has encouraged studies into the pathogenesis of this organism[5]. One of the challenges that face a diagnostic virology laboratory is a balance between rapid detection, structural preservation, and the appropriate fixation and inactivation of pathogens. For rapid detection of new and unknown pathogens negative staining is still the best technique. However, for subsequent structural investigation, cryo-electron microscopy (cryo-EM) can provide a wealth of new information such as three-dimensional volumetric data. The enveloped viruses are particularly challenging to work with. They are sensitive to the; mechanical, chemical, and radiological treatments used in their purification and inactivation. In this abstract we present our preliminary findings that we have obtained on the structure of the Ebola virus.

We have grown the Zaire strain of Ebola virus in containment, and purified it by centrifugation. The virus was fixed, dialysed, and subsequently imaged in a FEI Tecnai $20 \mathrm{TEM}$ at $200 \mathrm{kV}$. In this investigation we first stained the virus with methylamine tungstate to ensure the integrity of the virus was preserved $(a, b)$. In all subsequent experiments Quantifoil grids (Quantifoil Micro Tools $\mathrm{GmbH}$ ) with $2 \mathrm{~m}$ holes were treated with glow discharge prior to virus adsorption. Specimens were freeze plunged using a Vitrobot Mark IV (FEI Company), with the temperature set at $4^{\circ} \mathrm{C}$, and the humidity set at $100 \%$. Frozen hydrated specimens were placed in a CT3500TR single tilt rotation liquid nitrogen cryo-transfer holder (Gatan, Inc.). Once in the microscope all image data was acquired using a low dose unit, and digital images were recorded with a $4 \mathrm{~K}$ Eagle CCD camera (FEI Company).

Initial cryo-images revealed the improved sample preservation obtained by using cryo-EM, compared to negative staining (a-d). The contrast in the fully hydrated cryo-EM sample is attributable to both internal and peripheral specimen mass. In comparison the negative stained sample is dehydrated, with surface staining and differential stain penetration into the interior of the virus. Cryo-EM images showed the Ebola virus has a diameter of $120 \mathrm{~nm}$ (including spikes), and $100 \mathrm{~nm}$ (envelope only). The virus has a central nucleocapsid with a "haring bone" appearance. The nucleocapsid is $30 \mathrm{~nm}$ in diameter, with a 6-7 $\mathrm{nm}$ pitch. Glycoprotein spikes are clearly visible on the viral envelope; the spikes extend $\sim 10 \mathrm{~nm}$ from periphery of envelope. These results help to provide insight into the ultrastructure of this rare and extremely dangerous virus, for which there is still little structural data available. Cryo-electron tomography research is presently underway to further advance our knowledge of Ebola virus. 
References

[1] J.S. Towner et al., PLoS. Pathog., 4 (2008), e1000212.

[2] C.J. Peters and J.W. LeDuc, J. Infect. Dis., 179 Suppl 1 (1999), ix.

[3] H. Feldmann et al., Nat. Rev. Immunol., 3 (2003), 677.

[4] A. Sanchez et al., J. Gen. Virol., 73 (Pt 2) (1992), 347.

[5] M. Bray, Antiviral Res., 57 (2003), 53.
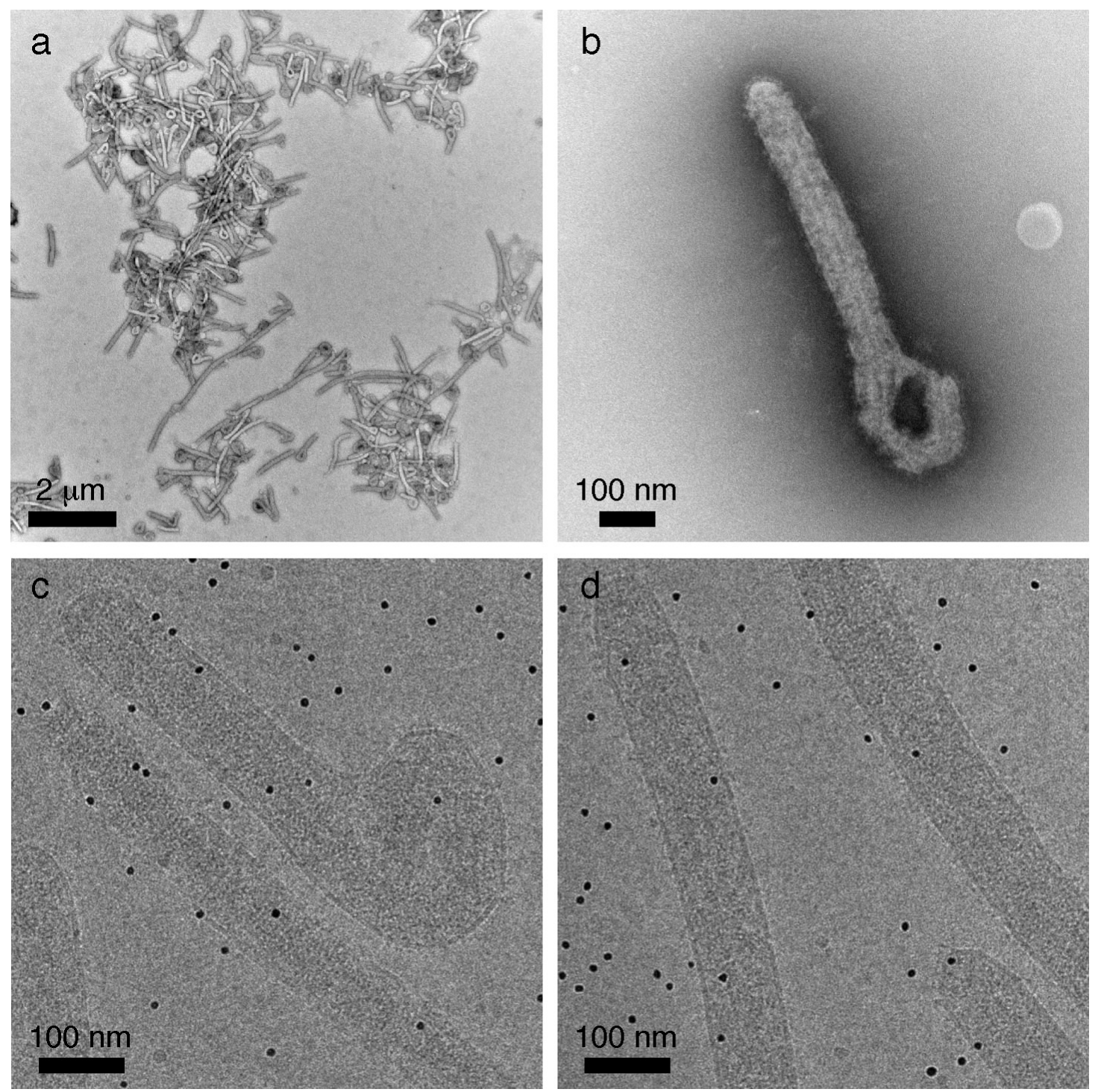

Images of the Ebola virus. (a,b) Ebola virus negatively stained with methylamine tungstate. These images showed that the virus was intact after purification and fixation. (c,d) Frozen hydrated images of the Ebola virus. The specimens were prepared with $10 \mathrm{~nm}$ colloidal gold which were used for automated focusing, and subsequent tomographic analysis. 